Commentary/Thomas \& Karmiloff-Smith: Are developmental disorders like cases of adult brain damage?

the endpoint of development are likely to be the outcome of an extended atypical developmental trajectory, determined by the initial structural anomalies in the system and by its atypical interaction with the environment (Karmiloff-Smith 1997; 1998). This is a plausible position in part because current genetic and biological understanding favors the view that in disorders of a genetic origin, the effects will be wide spread and nonspecific, and thus result in brain changes that are likely to affect learning and development. Developmental cognitive neuropsychology has not drawn a sufficiently clear distinction between acquired deficits and congenital ones, and has too often applied similar theoretical frameworks to both, disregarding the crucial effects of development.

The question that I would like to raise relates to the above position. The assumption that in cases of congenital disorders of genetic origin the child in fact learns with a different brain, predicts that disordered populations will more often than not exhibit an abnormal developmental course. However, it is rather rare to encounter reports of performance in disordered populations that is of a kind never seen in typically developing children. One typically sees reports of delays and asynchronous development, but rarely have truly deviant patterns been reported. A typical example concerns the notion of extended optional infinitive, describing asynchronous development in children with Specific Language Impairment (SLI) (Rice et al. 1998). Reports of developmental delays in neurodevelopmental disorders of genetic origin are too numerous to quote.

Especially problematic in this regard are claims based on group results when the comparisons are drawn between group means and prevalent tendencies. For example, consider the claim made by Cossu Rossini and Marshall (1993) that children with Down syndrome read without phonological awareness. This work might have qualified as an example of an atypical learning trajectory. However, the participants with Down syndrome did not perform at zero level. Rather, in some cases they scored 13 out of 21 correct, and there was just one child who scored 0 on three out of four tasks (Bryne 1993). Hence, whereas there were statistically significant differences between children with Down syndrome and typically developing children on phonological awareness tasks, I doubt whether one can attribute these differences to a different learning trajectory.

The authors cite work on face processing in Williams syndrome suggesting that, despite normal face recognition abilities, the underlying processes by which resolutions are arrived at is atypical (Deruelle et al. 1999). But are these processes of the kind never encountered in children with normal brains? In a similar vein, in all of the research on language in Williams syndrome there have been very few reports, if any at all, of errors that are never seen in normal children (but see Capirci et al. 1996). Most of the findings concern delayed acquisition, higher error rates, and either less (Karmiloff-Smith et al. 1997) or more (Clahsen \& Almazan 2001) control over grammatical rules relative to memorized words. The conclusion from most of the work on Williams syndrome - and the authors' work is no exception - as well as from work on other developmental syndromes, is that there are set paths for language learning even under pathology, with variation in speed and onset of various components, yet with minimal options for deviation that are not time-related (Levy et al. 2000).

This is perhaps not surprising given the plasticity that is inherent in the young brain. Brain plasticity has been invoked mostly in cases of focal damage (cf. Bach 1990). However, if brain plasticity is assumed in congenital disorders of genetic origin, then the expectation is that despite genetic abnormalities that cause structural deviations from typicality, functional normalcy may be preserved throughout development. In fact, the question is to what extent structure equals function in the brain and, consequently, whether structural differences, such as are seen in genetic disorders, predict functional differences. The fact that there are healthy children who pursue developmental trajectories that characterize children with abnormal brains, suggests that these atyp- ical developmental options are not a consequence of structural abnormality, and that they may operate in normalcy too. In conclusion, whether structure equals function in the brain is an empirical question that has to be addressed in reference to specific developmental domains. Theoretically, however, given that there is plasticity, the fact that there is structural abnormality does not in itself predict functional abnormality.

\section{Coconstructed functionality instead of functional normality}

\author{
Shu-Chen $\mathrm{Li}^{\mathrm{a}}$ and Ulman Lindenberger ${ }^{\mathrm{b}}$ \\ ${ }^{a}$ Center for Lifespan Psychology, Max Planck Institute for Human \\ Development, D-14195 Berlin, Germany; bS School of Psychology, Saarland \\ University, D-66041 Saarbrücken, Germany. shuchen@mpib-berlin.mpg.de \\ lindenberger@mx.uni-saar land.de http://www.mpib-berlin.mpg.de \\ http://www.uni-saarland.de/fak5/lindenberger/members/ulman.htm
}

Abstract: We agree with the critique of the Residual Normality assumption. Moreover, we challenge monolithic views of functional normality. Throughout life, development and adaptation require variations in cortical functional circuitry within and across individuals. We propose the principle of "coconstructed functionality" which maintains that brain-behavior functional correspondences are dynamically coproduced by neurobiological, experiential, and contextual processes.

Thomas \& Karmiloff-Smith (T\&K-S) argue against a canonical hypothesis of developmental cognitive neuropsychology. According to this hypothesis, similarities between domain-specific cognitive impairments observed after damage to the adult brain, on the one hand, and developmental disorders, on the other, point to similarities in underlying causes, thereby lending support to a modular organization of normal adult cognition. They challenge the validity of this inference by demonstrating that its underlying assumption (the "Residual Normality assumption") is untenable, primarily because developing systems generally compensate for selective deficits by modifying other parts of the system. We agree with this critique and would like to take it a step further. We call into question the notion of functional normality and advocate the principle of "coconstructed functionality" for investigating individual and life-span differences in brain-behavior mappings.

Coconstructed functionality. A monolithic divide between normality and abnormality is unproductive and misleading, if "normal" signifies invariance of functional correspondences between brain and behavior. Variations within and between individuals are fundamental to living organisms (Ford 1987; Mayr 1998). Rather than being one-to-one, functional mappings of brain structures and processes to behaviors tend to be many-to-many. At the behavioral level, research on the organization of intellectual abilities has long since identified substantial individual differences within the normal range of functioning (e.g., Deary 2001; Spearman 1904). Similarly, life-span intellectual development is conceptualized as dynamic, multifunctional, and multidirectional (e.g., Baltes et al. 1998; Cattell 1971; Engle 2002; Horn 1968). Among others (e.g., Siegler 1994), Francophone scholars emphasize the importance and ubiquity of multiple developmental pathways with vicariance (i.e., alternation among several processes fulfilling similar functions) being a source of within- and between-individual variations and development (Lautrey, in press; Reuchlin 1978; de Ribaupierre 1993). At the neurobiological level, intra-individual (e.g., Makeig et al. 2002), interindividual (e.g., Toga 2002), and life-span (e.g., Cabeza 2002; Johnson 2001; Raz 2000) differences in brain functioning are large and widespread in the absence of pathology (e.g., within the "normal range" of functioning). Cognition and behavior reflect the reciprocal interactions between neurobiological and experiential contexts of life that vary both within and across individuals. Therefore, instead of reaffirming the orthodox dichotomy between the normal 
and the abnormal, "normality" is better viewed as the potential of individuals (and environments) to produce an adaptive range of behaviors in a variety of ways. Specifically, any attempt to define normality in terms of invariant brain-behavior mappings neglects the pivotal role of variation at all level and timescales of biological (Mayr 1998) and biocultural organization (Baltes \& Singer 2001; Li 2003).

Life-span differences in brain-behavior mappings. In arguing against biological determinism, recent coconstructive theories (e.g., Baltes \& Singer 2001; Cole 1999, Elman et al. 1996; Li 2003; Nelson 1996) have stressed the importance of interactive processes and developmental plasticity occurring across different timescales and levels in shaping brain-behavior relations. For example, face processing is less localized or specialized in infants than in adults. In infants, face processing involves both left and right ventral visual pathways; in adults, face processing primarily involves the right ventral visual pathway (de Haan et al. 2002). During senescence, cortical information processing in different brain areas becomes less specific or more integrated (see Cabeza 2002; Logan et al. 2002; Reuter-Lorenz 2002 for reviews). A recent cross-level neurocomputational theory suggests that senescent decrements in neuromodulation, which affect the distinctiveness of neuronal representations of environment-experience interactions, may account for some of the observed losses in cortical specialization (e.g., Li et al. 2001). It is unclear to what extent cortical reorganization in late adulthood directly reflects senescent changes in structural and neurochemical integrity, or compensatory adaptations to such changes. In either case, evidence from both child development and aging indicates that the modal functional circuitries brought to bear upon "nominal cognitive tasks" differ widely by age.

At the behavioral level, life-span changes in the structure of intelligence support our critical appraisal of functional normality. The differentiation-dedifferentiation hypothesis (e.g., Baltes et al. 1980) postulates that intellectual abilities are rather undifferentiated in childhood, undergo differentiation during maturation, leading to a multifaceted ability structure that stays largely invariant during adulthood, and again become more homogeneous (dedifferentiated) during aging. A recent study with a wide range of ages (6 to 89 years) and a comprehensive battery of intelligence tests and standard reaction-time tasks revealed that correlations among different aspects of intelligence and information-processing parameters are indeed higher at both ends of the life span $(\mathrm{Li}$ et al., in press). Again, such life-span transformations call for dynamic views on brain-behavior mappings.

Individual differences in brain-behavior mappings. Variability and plasticity of functional circuitry are not confined to the extreme ends of the life span; rather, they are general prerequisites for cumulative experiential tunings reflecting the provisions, demands, and constraints of specific sociocultural contexts. For example, posterior regions of the hippocampi, involved in processing spatial representation of the environment, have been reported to be significantly larger in London taxi drivers than in age-matched controls (Maguire et al. 2000). Similarly, there are marked differences between native speakers of English and Italian with respect to the brain areas involved in reading (Paulesu et al. 2000): English readers show greater activations in the left posterior inferior temporal gyrus and the anterior frontal gyrus, areas associated with word retrieval. Presumably, these differences reflect language-specific adaptations in the functional circuitry supporting grapheme-to-phoneme translation.

Conclusion. Life-span changes, interindividual differences, and intra-individual variability in brain-behavior mappings are not adequately described or explained in relation to a "gold standard" of normality. The principle of coconstructed functionality, with its emphasis on socioculturally embedded experiential tuning, seems more productive than the normal/abnormal dichotomy. To capture the dynamic and multivariate picture of brain-behavior relations, we need systematically to investigate biocultural coconstructive processes that lead to individual and life-span differences in cortical functioning. Undoubtedly, invariant properties of cortical organization will also emerge from such an approach, but they will be located at higher levels of abstraction, be it coherent patterns of neural assembly (e.g., Singer 1995), or general principles of human behavior (e.g., Nesselroade 1991).

\section{ACKNOWLEDGMENT}

We thank Paul B. Baltes for his comments on an early draft of the commentary.

\section{What can developmental disorders tell us about modularity?}

\section{Gary F. Marcus \\ Psychology Department, New York University, New York, NY 10012. gary.marcusl@nyu.edu http://www.psych.nyu.edu/gary/}

Abstract: This commentary discusses the logic of inferring modularity or the lack of modularity from observed patterns of developmental disorders.

Developmental disorders are, as Thomas \& Karmiloff-Smith (T\&K-S) observe, not necessarily like cases of adult brain damage. Their simulations well illustrate why one cannot directly infer underlying mental organization from surface behavior, a problem that has long made the discipline of psychology challenging (Chomsky 1959; McClelland 1979). And T\&K-S are quite right to highlight the perils of inferring modularity from a pairing of apparently normal behavior in some domains with impaired behaviors in other domains; seemingly normal behavior could always be the product of compensatory mechanisms. (Exactly the same worry hampers the study of second-language acquisition - when a nonnative speaker succeeds, it is difficult to know in advance whether she is relying on remnants of universal grammar or some kind of domain-general cognitive substitute.)

But the naive inference from impairment in multiple domains to a lack of modularity is equally riddled with danger. Although cognitive systems are often caricatured as being the product of either entirely modular or entirely shared resources, the reality is that every complex neural system - even ones that are modular in the sense of performing computations that are informationally encapsulated - probably relies on a mixture of domain-specific and domain-general resources. A system for language understanding, for example, probably relies on both shared resources like memory and symbol-manipulation machinery (Marcus 2001) and nonshared machinery that is specialized for linguistic representation (Hauser et al. 2002). A disorder that impaired the neural underpinnings of memory might well then impair both linguistic and nonlinguistic systems, even if the linguistic system included some specialized resources. Disorders that affect multiple domains hence may tell us little about questions about underlying modularity.

What is true at the psychological level is perhaps even more true at the genetic level. Even where two organs are specialized and physical distinct, they often depend in large part on overlapping sets of genes. A chicken's wing and its leg, for example, are for the most part built under the direction of the same set of genes. Out of the hundreds or thousands of genes involved in limb formation, only a handful style $=^{\prime}$ color:black' $>$ (Margulies et al. 2001), black' > . In a similar way, it is possible, perhaps even likely, that separate style $=^{\prime}$ color:black' $>$ on common sets of genes, just as separate limbs the case of the specialization of limbs, the specialization of neural function might rely on a handful of novel genes specific to that domain interacting with a larger number of conserved (shared) genes.

It follows that we must be extremely careful about inferring a lack of modularity from the distribution of disorders. We should expect that many disorders will affect multiple subsystems, yet keep our eyes open for deficits that are more focused. Even if, for 